\title{
A 30-year-old woman with vision loss and painful eye movements
}

\author{
Jonathan A. Micieli MD CM, Edward Margolin MD
}

A 30-year-old woman presented to the emergency department with a two-day history of pain in her right eye that was worse with eye movements. She also complained of blurred vision in the right eye. She had no floaters or eye redness and did not have recent trauma. Her ocular and medical history were unremarkable, and she was not taking medications.

On physical examination, the patient appeared well and had normal vital signs. Her best corrected visual acuity was 20/50 in the right eye and 20/20 in the left eye, and the intraocular pressures were normal at $13 \mathrm{~mm} \mathrm{Hg}$. The patient had a relative afferent pupillary defect in her right eye, and her extraocular movements were full. There was no proptosis or periorbital swelling. Findings on slit-lamp examination were normal, and funduscopic examination with pupil dilation showed a normal macula, peripheral retina and optic nerve. Results of a full neurologic examination were otherwise normal.

\section{What is the most appropriate next step?}

a. Order complete blood count, antinuclear antibody test and venereal disease research laboratory test

b. Diagnose optic neuritis and arrange for nonurgent magnetic resonance imaging (MRI) of the brain with ophthalmology follow-up

c. Obtain computed tomography of the orbits to rule out compressive lesion of the optic nerve

d. Perform lumbar puncture to rule out infectious causes and for oligoclonal band screening of the cerebrospinal fluid (CSF)

The answer is (b). Optic neuritis is an inflammatory, demyelinating disease of the optic nerve typically diagnosed on clinical grounds without neuroimaging. (By convention, the term "optic neuropathy" will be used in this article when the cause of inflammation of the optic nerve is not demyelination.) Laboratory and imaging studies may be used for prognostic purposes, but they are not required. As was the case with this patient, the typical person with optic neuritis is young (mean age $32 \mathrm{yr}$ ), female (3:1 ratio of women to men) and white (> 75\% of patients). ${ }^{1}$

Patients present with subacute loss of vision that develops over hours or days. In most instances, the vision loss is associated with orbital pain (in 92\%) that is worse with eye movements (in 87\%). ${ }^{1}$ The pain is thought to be due to mechanical irritation of the inflamed optic nerve. ${ }^{2}$ About one-third of patients also note photopsia. ${ }^{1}$

An important finding on physical examination is a relative afferent pupillary defect, which is always seen in optic neuritis except in the rare instance of symmetric bilateral disease. Other important findings on examination are decreased central visual acuity (although all ranges of vision from 20/15 to no light perception may be present) and edema of the optic nerve head (in one-third of patients), which was not seen in this patient. ${ }^{1}$

The clinical data for the diagnosis and treatment of optic neuritis are derived from a welldesigned randomized controlled trial: the Optic Neuritis Treatment Trial. ${ }^{1}$ The trial enrolled more than 400 consecutive patients aged $18-45$ years from multiple sites across North America who had an acute clinical syndrome consistent with unilateral optic neuritis and whose visual symptoms were present for up to eight days. ${ }^{1}$

Optic neuritis is diagnosed when patients who fit the demographic pattern present with typical symptoms (i.e., acute to subacute decrease in vision and eye pain that is worse with extraocular movements) and findings on physical examination (i.e., relative afferent pupillary defect with or without optic nerve edema). Reduced colour vision and visual field defects may be seen on formal testing.

Atypical cases that do not fit the usual demographic pattern or clinical presentation require a more urgent referral to an ophthalmologist to assess for other causes of vision loss. The differential diagnosis includes ischemic optic neuropathy,
Competing interests: None declared.

This article has been peer reviewed.

Correspondence to: Jonathan Micieli, jonathan .micieli@utoronto.ca

CMAJ 2015. DOI:10.1503 /cmaj.141389 
infectious optic neuropathy (e.g., due to syphilis), compressive lesion of the optic nerve and infiltrative disease such as sarcoid or central nervous system lymphoma.

In the Optic Neuritis Treatment Trial, all patients received the fluorescent treponemal antibody absorption test, and none was found to have active syphilis as the cause of optic neuritis. ${ }^{1}$ Similarly, antinuclear antibody testing was noncontributory, which makes answer (a) less likely. A person with a compressive lesion of the optic nerve (c) usually presents with gradual vision loss and, depending on the location of the lesion, may have other associated signs such as proptosis or optic nerve edema or pallor, which was not seen in this patient. Moreover, lumbar puncture was performed in $31.5 \%$ of the patients in the Optic Neuritis Treatment Trial and did not reveal an additional cause for the vision loss other than demyelinating disease. ${ }^{1} \mathrm{Al}-$ though CSF oligoclonal bands may be seen in some patients with multiple sclerosis (MS), this test (d) is not indicated for a patient with a first presentation of typical optic neuritis.

\section{How do you respond when the patient asks if she has multiple sclerosis?}

a. Multiple sclerosis eventually develops in all patients with optic neuritis and is most often diagnosed after five years

b. Optic neuritis is only rarely associated with MS

c. Magnetic resonance imaging of the brain is the best way to determine MS risk, and a more accurate prognosis can be given after the MRI results

d. The diagnosis of MS is almost certain, and disease-modifying therapy should be started

The answer is (c). The strongest predictor of MS is the presence of white matter lesions. The Optic Neuritis Treatment Trial found that, among patients with at least one white matter lesion of at least $3 \mathrm{~mm}$ in diameter on $T_{2}$-weighted MRI sequence, the 15 -year risk of clinically definite MS was $72 \% .^{3}$ Among those who had no lesions, the 15 -year risk was $25 \%$. To meet MS diagnostic criteria in the trial, patients had to have a clinical examination documenting a second new neurologic deficit attributable to demyelination that was consistent with neurologic symptoms, lasted at least 24 hours and occurred at least four weeks after the initial optic neuritis event. ${ }^{3}$ Recurrent episodes of optic neuritis in either eye were not considered in the diagnostic criteria for MS, because demyelinating events need to be separated both in space and time.
Having optic neuritis as the first manifestation of MS is considered a favourable prognostic factor. ${ }^{4}$ Among the patients in the Optic Neuritis Treatment Trial who subsequently received a diagnosis of clinically definite MS, there was a relatively low frequency of substantial disability (the Expanded Disability Status Scale score at 10 -year follow-up was $<3$ in $65 \%$ of patients and $>6$ in $17 \%)^{5}$

\section{How do you respond when the patient asks if her vision will return and if she needs treatment?}

a. Unfortunately, the vision will not recover to a great extent, and treatments are still in the experimental phase

b. The vision will return only if prompt treatment with intravenous corticosteroids is started

c. The vision will improve minimally over the next six months; no treatment is needed

d. The vision will most likely return close to baseline, and treatment with intravenous corticosteroids will only hasten visual recovery

The answer is (d). The typical course of visual recovery begins at two weeks, peaks at one month and continues up to one year after symptom onset. ${ }^{6}$ Most patients (95\%) experience a return of their central visual acuity back to baseline, although many still feel that their vision is "faded" or has diminished contrast. ${ }^{6}$ The course of visual recovery is important in confirming the diagnosis of optic neuritis; therefore, patients should be followed at regular intervals. Patients whose vision does not recover require further investigations for other causes of vision loss.

The Optic Neuritis Treatment Trial found that intravenous corticosteroid treatment with methylprednisolone $(1 \mathrm{~g} / \mathrm{d}$ for $3 \mathrm{~d})$ followed by oral prednisone treatment $(1 \mathrm{mg} / \mathrm{kg}$ for $11 \mathrm{~d})$ given within 8 days after onset of symptoms improved the speed of visual recovery by about 4 to 6 weeks but had no effect on visual outcomes at 6 months to 10 years. ${ }^{7}$ Oral corticosteroid treatment $(1 \mathrm{mg} / \mathrm{kg}$ for $14 \mathrm{~d})$ was found to be ineffective and increased the risk of new episodes of optic neuritis.?

\section{Discussion}

Optic neuritis is a demyelinating inflammatory condition most often seen in young white women. Optic neuritis is the presenting feature of MS in $15 \%$ to $20 \%$ of patients and occurs in $50 \%$ at some time during the course of their illness. ${ }^{8}$ The incidence is highest in populations 
located at higher latitudes, such as the northern United States and Canada, and is lowest in regions closer to the equator. ${ }^{9}$ The pathophysiology is similar to that of MS and is thought to be immune-mediated.

Optic neuritis is a clinical diagnosis that does not require laboratory findings or neuroimaging to make a diagnosis. Patients should be followed at regular intervals to ensure that they follow the typical course of visual recovery and to assess for new neurologic symptoms. Patients should be reassured that a diagnosis of optic neuritis does not necessarily mean that MS will develop and that if MS does develop, there is a good chance it will follow a more benign course.

\section{References}

1. Optic Neuritis Study Group. The clinical profile of optic neuritis: experience of the Optic Neuritis Treatment Trial. Arch Ophthalmol 1991;109:1673-8.

2. Fazzone HE, Lefton DR, Kupersmith MJ. Optic neuritis: correlation of pain and magnetic resonance imaging. Ophthalmology 2003;110:1646-9.

3. Optic Neuritis Study Group. Multiple sclerosis risk after optic neuritis: final Optic Neuritis Treatment Trial follow-up. Arch Neurol 2008;65:727-32.

4. Runmarker B, Andersen O. Prognostic factors in a multiple sclerosis incidence cohort with twenty-five years of follow-up. Brain 1993;116:117-34.

5. Beck RW, Smith CH, Gal RL, et al.; Optic Neuritis Study Group. Neurologic impairment 10 years after optic neuritis. Arch Neurol 2004;61:1386-9.
6. Optic Neuritis Study Group. Visual function 15 years after optic neuritis: a final follow-up report from the optic neuritis treatment trial. Ophthalmology 2008;115:1079-82.e5.

7. Beck RW, Cleary PA, Anderson MM Jr, et al.; Optic Neuritis Study Group. A randomized, controlled trial of corticosteroids in the treatment of acute optic neuritis. N Engl J Med 1992;326:581-8.

8. Balcer LJ. Clinical practice. Optic neuritis. N Engl J Med 2006; 354:1273-80.

9. Percy AK, Nobrega FT, Kurland LT. Optic neuritis and multiple sclerosis: an epidemiologic study. Arch Ophthalmol 1972;87:135-9.

Affiliation: Department of Ophthalmology and Vision Science, University of Toronto, Toronto, Ont.

Contributors: Both authors contributed equally to the writing and revising of the manuscript, accepted the final version submitted for publication and agreed to act as guarantors of the work.

$C M A J$ invites submissions to "What is your call?" Clinical details (including images) are presented with a multiple-choice question about the diagnosis. The answer and a brief discussion of the condition follow. We specifically invite submissions illustrating common or important radiographic and electrocardiographic diagnoses of appeal to a general audience. We require authors to obtain consent from the patient for publication of his or her story (form available at www.cmaj.ca/site/authors/checklist.xhtml). Submit manuscripts online at http://mc.manuscriptcentral .com/cmaj.

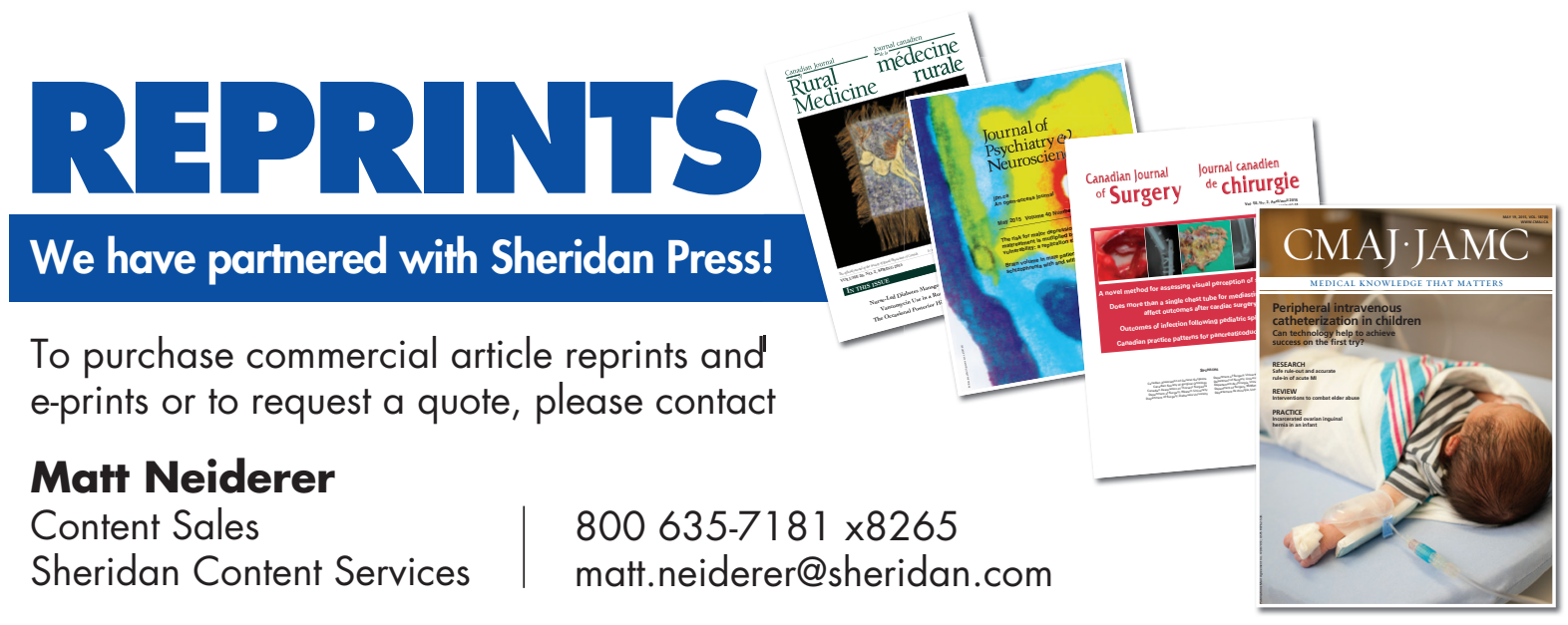

